Recherches en didactique des langues et des cultures

Les cahiers de l'Acedle

18-1 | 2021

En quoi les langues ont-elles un rôle à jouer dans les sociétés mondialisées au sein d'une Europe fragilisée ?

\title{
Prescriptions et pratiques dans l'enseignement des langues en Europe centrale et orientale
}

Focus sur le contexte roumain

Veronica Hagi et Monica Vlad

\section{(2) OpenEdition}

Journals

Édition électronique

URL : https://journals.openedition.org/rdlc/8575

DOI : $10.4000 /$ rdlc. 8575

ISSN : 1958-5772

Éditeur

ACEDLE

Référence électronique

Veronica Hagi et Monica Vlad, «Prescriptions et pratiques dans l'enseignement des langues en

Europe centrale et orientale », Recherches en didactique des langues et des cultures [En ligne], 18-1 |

2021, mis en ligne le 03 mai 2021, consulté le 03 août 2021. URL : http://journals.openedition.org/ rdlc/8575; DOI : https://doi.org/10.4000/rdlc.8575

Ce document a été généré automatiquement le 3 août 2021.

\section{(2) $(\oplus \otimes \Theta$}

Recherches en didactique des langues et des cultures is licensed under a Creative Commons AttributionNonCommercial-NoDerivatives 4.0 International License 


\section{Prescriptions et pratiques dans l'enseignement des langues en Europe centrale et orientale}

Focus sur le contexte roumain

Veronica Hagi et Monica Vlad

1 Ancien centre géographique et point d'ancrage historique de l'Europe, l'Europe centrale et orientale semble être entrée dans une zone d'ombre et de silence depuis que la plupart des pays qui la composent sont devenus membres de l'Union Européenne. Comme si les traditions historico-politiques de cette partie de l'Europe, marquées très souvent par le poids excessif du contrôle communiste, pouvaient être uniformisées par l'adhésion aux valeurs et aux concepts partagés depuis longtemps déjà par les pays ayant adhéré il y a plus longtemps aux institutions européennes. Du point de vue de l'étude des langues, l'uniformisation dont on vient de parler met entre parenthèses certains points-clés que nous aimerions mettre en débat ci-dessous.

Dans un numéro de Synergies Roumanie paru en 2009 et qui reste l'une des références en la matière, le dossier thématique La didactique du français dans les pays de l'Europe centrale et orientale (Vlad dir., 2009) propose des regards complémentaires de didacticiens et d'enseignants provenant de Roumanie, Pologne, Croatie et Albanie sur l'évolution de la recherche et de la profession didactique dans le domaine des langues depuis l'intégration de ces pays dans la dynamique européenne. Reprenons d'abord quelquesuns des éléments transversaux qui apparaissent de manière récurrente dans leurs propos afin de voir, par la suite, où en est la situation dix années plus tard.

3 Le premier point à souligner tient à l'obligation unanimement ressentie par les auteurs d'alignement des programmes nationaux sur les orientations européennes après 1990 et sur les prescriptions du CECR après 2000. Comme l'explique Dedja (ibid. : 55) :

«l'adhésion de l'Albanie à des organismes internationaux a rendu nécessaire l'application de réformes dans le système éducatif, l'élaboration d'un nouveau curriculum national [...]. Les textes sont rédigés conformément au Portfolio des langues et au CECR ». 
Cette nécessité n'est souvent pas même questionnée. Ministères de l'Enseignement, universitaires, didacticiens et enseignants reçoivent les nouveaux textes européens comme une aubaine après les années d'isolement subis à cause des différents régimes autoritaires.

5 Ceci dit, l'imposition est regardée d'un œil critique pour au moins deux aspects : le manque d'explicitation des documents officiels qui se sont alignés sur les prescriptions européennes en mettant sous silence les présupposés théoriques qui les sous-tendaient, et leur difficile application sur le terrain de la classe. Comme le dit Nasta (ibid. : 92) :

«d'une part, la tentation est grande de placer la barre haut en adoptant les référentiels européens [...]. D'autre part, bon nombre des entrées nouvelles (la compétence de transfert, la compétence de médiation, l'entrée culturelle et interculturelle, les nouveaux modes d'évaluation, l'usage des portfolios, le recours souhaitable à la pédagogie des projets) ne bénéficient [dans le contexte roumain] ni d'une banque d'aide ni d'un appareil minimal d'explication/illustration. »

6 Cette stratégie « de l'implicite » ou « de l'esquive » (Nasta, 2009: 94, 95) est également dénoncée lorsqu'il s'agit des pratiques de classe :

« la mise en place trop rapide du processus de Bologne, les modifications constantes des programmes d'enseignement désorientent les étudiants et les enseignants. » (Vishkurti et Ben-Nacer, 2009 : 106, 107).

7 Les années de paralysie communicative imposés par le communisme ne rendent pas facile la tâche des enseignants qui ne savent / ne peuvent / ne veulent pas enseigner l'oral communicatif, le travail par projets autour d'une tâche de communication, etc.

8 Un autre élément transversal qui ressort de la plupart des textes réunis dans le numéro de 2009 de Synergies Roumanie tient à l'importance des organismes internationaux dans l'accompagnement de la formation des enseignants aux orientations méthodologiques communicatives :

«[Dans] la formation continue des enseignants déjà en place, [...] des organismes tels l'AUPELF et ensuite l'AUF jouent un rôle important, relayé au niveau européen par une politique active du Conseil de l'Europe - pour ne citer que les activités du CELV de Graz » (Cosaceanu ibid., p. 24)

«Un [...] élément positif est le plan de promotion de la langue française lancé par l'Ambassade de France en Pologne, en coopération avec l'Association des Professeurs de Français de Pologne - Prof-Europe » (Cichon ibid., p, 51)

9 Ces remarques mènent vers deux conclusions: les organismes de formations francophones et européens sont appelés par les enseignants et les chercheurs d'une part à expliciter les orientations méthodologiques restées implicites lors de leur transfert trop rapide vers les contextes des pays du centre de l'Europe et, d'autre part, à légitimer des pratiques didactiques nouvelles auprès de ceux qui se mettent dans la mouvance communicative et actionnelle sans aucune autre validation.

10 Enfin, le point fédérateur et qui semble être appelé de tous leurs vœux par tous les acteurs impliqués dans la rédaction des articles, tient à la promotion de la recherche didactique dans le contexte central européen, pan de réflexion en l'absence duquel toute réforme durable ou tout changement de paradigme serait vide de sens et n'aurait pas de chance d'aboutissement. Dans l'Introduction du numéro de Synergies Roumanie de 2009, l'Attachée de coopération éducative du Service de coopération et d'action culturelle de l'Ambassade de France en Roumanie cite à plusieurs reprises la recherche didactique :

- en tant que première direction à suivre pour moderniser les cursus : 
« introduire la recherche didactique au cœur de la formation initiale et continue des professeurs de français avec un nouveau modèle à établir » (p.16)

- en tant que priorité conditionnant le développement de l'enseignement du français en Roumanie :

« il convient donc pour assurer au français sa place en Roumanie d'en faire une discipline nourrie par les recherches en cours, à leur tour terreau de nouveaux professeurs et formateurs» $»$ (p.17).

11 Qu'en est-il dix années plus tard ? Nous allons formuler quelques points de vue à partir de l'examen cette fois-ci du contexte roumain, que nous maitrisons le mieux. Pour ce qui est, d'abord, de la longévité des prescriptions européennes en matière d'enseignement des langues, elle est évidente. Les nouveaux programmes pour le collège rédigés en 2017 proposent un traitement unifié des langues maternelles et étrangères et s'appuient de manière explicite sur les documents suivants: Key Competences for Lifelong Learning - a European Reference Framework, et le CECR (http:// programe.ise.ro/Portals/1/Curriculum/2017-progr/19-Limba \%20moderna1.pdf)

Pour ce qui est des grandes instances linguistiques internationales impliquées dans la promotion et dans la diffusion des langues, on constate un désistement progressif de leur part dans le contexte roumain (et plus largement centre-européen), désistement accompagné, de manière évidente, d'une diminution des moyens mis à disposition. Ce vide qui est laissé derrière ne peut pas être comblé de soi, tout au moins pas dans un pays avec des traditions de centralisation fortes et avec des moyens encore peu alignés sur ceux qui sont disponibles en Europe Occidentale.

13 Enfin, la masse critique représentée par ceux qui avaient participé à l'introduction dans les pays d'Europe centrale et orientale des textes européens s'est amenuisée au fil du temps: non pas parce que l'application des réformes est devenue plus évidente et moins problématique, mais parce que la formation et la réflexion en matière de didactique des langues a été reléguée dans des départements universitaires séparés des Facultés de langues étrangères et a perdu de sa légitimité - tout au moins dans le contexte roumain.

Les trois grandes catégories de chercheurs en didactique que l'on peut identifier actuellement en Roumanie (les didacticiens à proprement parler, les universitaires linguistes et litteraires et les enseignants du pré-universitaire) ont du mal à être légitimées en l'absence d'une reconnaissance nationale de leur existence et activité. Cantonnées dans les limites d'une formation philologique traditionnelle, la même qu'il y a quarante ans, les universités ont du mal à prendre en compte les déplacements de paradigme préconisés par les méthodologies communicatives et actionnelles. Les cours de didactique sont encore très souvent pris en charge par des linguistes ou des littéraires plus préoccupés par la transposition de leurs recherches que par ce qui se passe réellement dans les classes ou par les problématiques et réflexions qui traversent actuellement le champ de la DDL. Reconnu et déploré, ce problème lié au statut problématique et peu légitimé de la recherche et de la formation didactiques en Roumanie a déjà fait l'objet d'analyses (Vlad, 2019). Il a pourtant peu été mis en relation avec les caractéristiques historico-culturelles du contexte qui, marqué par les longues années de communisme, ne peut pas passer du jour au lendemain aux préconisations européennes en matière d'enseignement des langues, tout au moins pas dans un contexte dans lequel seules la linguistique et la littérature ont leurs lettres de noblesse dans la zone académique. Comme l'écrivait à juste titre Jean-Louis Chiss (2009 : 31) : 
la reconfiguration d'Humanités modernes au sein d'une "éducation européenne » n'est pas une entreprise aisée, car [...] on ne saurait oublier que cette éducation européenne a son historicité, celle de l'Europe centrale, des intellectuels tchèques, de Comenius à l'écrivain Jan Kott, pour qui elle voulait dire le passage par le nazisme et le communisme, les guerres, la Shoah et la Résistance.

15 A partir de ces éléments essentiels liés à l'historicité et à la contextualisation, la problématique de la réflexivité enseignante définie comme « le moteur d'un savoir agir en contexte, garante de contextualisation, mais aussi d'une capacité à faire face à la complexité » (Bretegnier, $2014: 275)$ représente, à notre sens, autant le point nodal que le plus grand défi posé devant tous les acteurs. Dans un pays à longue tradition de centralisation, dans lequel les instances nationales ont du mal à inscrire la didactique des langues dans la nouvelle configuration, plus complexe, des humanités modernes, développer la réflexivité enseignante serait le bouclier essentiel capable de minimiser les effets d'un applicationisme aveugle des prescriptions venues d'ailleurs et qui sont affichées comme des desiterata officiels.

Dans l'espace d'une seule génération, les enseignants formés dans la bonne vieille tradition philologique, à même de vous réciter des vers de Racine et de Corneille et de faire des exercices compliqués de traduction sont amenés à imaginer des stratégies d'approche des documents authentiques, à concevoir des tâches pour l'enseignement communicatif-actionnel de la langue, à entrainer les apprenants dans des débats oraux sur des sujets de conversation contemporaine... Ils travaillent souvent dans les mêmes établissements que des collègues plus jeunes, pour lesquels la tradition philologique ne veut plus rien dire et qui sont formés dans les nouvelles conditions du système européen, conditions souvent peu réglementées par les instances de formation locales qui ne laissent plus aux universités la mission de formation des enseignants de langues. Ils se retrouvent tous démunis devant les nouveaux repères : devant le changement de paradigme préconisé par les différentes approches par compétences dans le domaine des langues, approches qui mettent l'accent non plus sur les savoirs sur la/les langues, mais sur les enjeux de la transmission, leur pourquoi, où et comment, les enseignants d'aujourd'hui ont souvent du mal à réfléchir sur leur propre pratique, sur ses finalités, sur les nouvelles contraintes venant aussi bien des documents européens, que surtout des changement sociétaux, des nouvelles formes de mobilité, du nouveau marché des langues. Les uns parce qu'ils ont été formés dans des formations universitaires inscrites dans un monde et dans un mode de réflexion différents, les autres parce qu'ils ont été formés de manière trop rapide dans des formations de courte durée, souvent incohérentes, par des formateurs venus d'ailleurs, empressés à transmettre la bonne parole du renouvellement méthodologique sans suffisamment de réflexion sur le contexte d'action.

17 Au carrefour de tous ces éléments et de ces points de débat, la clé de voûte nous semble être la formation des enseignants. Cette formation se doit de prendre en compte non seulement les courants méthodologiques européens, mais elle devra aussi, dans un souci d'agir éthique, accorder de l'importance aux traditions et à l'histoire politiques et éducatives de ces pays qui, avant de faire partie de l'Europe communautaire, sont des pays avec un passé particulier et avec une tradition de recherche et d'enseignement marquée par des années d'isolement et de silence. 


\section{BIBLIOGRAPHIE}

BRETEGNIER Aude, 2014, « Une réflexivité éthique comme « macro-compétence » professionnelle des enseignants/formateurs en langue(s) : vers une conception plus opérationnelle », dans M. Causa, S. Galligani et M. Vlad (dir.), Formation et pratiques enseignantes en contextes pluriels, Paris, Riveneuve, p. 273-290.

CHISS Jean-Louis, 2009, « Les humanités entre langue, littérature et culture », dans Le Français aujourd'hui 167, Paris, Armand Colin, p. 27-33.

CICHON Maria, 2009, „La formation initiale des enseignants de FLE dans les universités polonaises : les enjeux théoriques et les savoir-faire pratiques", dans Synergies Roumanie n. 4, Revue du Gerflint, p. 45-52.

COSACEANU Anca, 2009, „Formation initiale des formateurs : la didactique du FLE dans les universités roumaines", dans Synergies Roumanie n. 4, Revue du Gerflint, p. 23-28.

DEDJA Lorena, 2009, „La formation des enseignants de français et les perceptions des étudiants albanais en didactique du FLE sur leur profession future - résultats d'une enquête", dans Synergies Roumanie n. 4, Revue du Gerflint, p. 53-58.

NASTA Dan Ion, 2009, „La compétence de lecture en FLE - un défi plutot mal relevé dans les textes officiels. Regards croisés sur les programmes d'études secondaires en Roumanie", dans Synergies Roumanie n. 4, Revue du Gerflint, p. 91-101.

VISHKURTI Silvana et BEN NACER Barbara, 2009, „Quelques reflexions sur la didactique des langues en Albanie", dans Synergies Roumanie n. 4, Revue du Gerflint, p. 103-109.

VLAD Monica, 2019, « La didactique du FLE en contexte roumain : une discipline en quête de légitimité ", dans Le Français dans le Monde, Recherches et applications n.66, Paris, CLE-International.

VLAD Monica (dir.), 2009, « Sciences du langage et didactique des langues. Frontières et rencontres ", Synergies Roumanie n. 4, Revue du Gerflint, https://gerflint.fr/Base/Roumanie4/ roumanie4.html, consulté le 31.03.2019.

\section{AUTEURS}

\section{VERONICA HAGI}

Université Ovidius, Constanta (Roumanie)

Veronica-Diana HAGI est enseignante de FLE à l'École Gymnasiale numéro 30 « Gheorghe ȚiȚeica » de Constanța et doctorante à l'École Doctorale de Sciences Humaines de l'Université « Ovidius » de ConstanȚa, collaboratrice au Département de Langues Modernes. Elle prépare une thèse dans le domaine de la sociolinguistique et de la didactique des langues intitulée Du répertoire langagier vers les représentations sur les langues par l'écriture autoréflexive. Elle a publié des articles sur le domaine large du plurilinguisme dans des réseaux nationaux et internationaux. Parmi ses domaines d'intérêt, nous retrouvons la littérature roumaine, la littérature comparée, la didactique du FLE, les approches plurielles dans la didactique des langues.

Courriel : veronicahagi[at]yahoo.com 


\section{MONICA VLAD}

Université Ovidius, Constanta (Roumanie)

Monica Vlad est professeure des universités à la Faculté des Lettres et dirige l'Ecole doctorale de Sciences Humaines de l'Université Ovidius de Constanta, Roumanie. Elle enseigne la linguistique et la didactique du français langue étrangère et du plurilinguisme et est impliquée dans de nombreux projets à envergure internationale. Ses recherches portent notamment sur la formation des enseignants dans le contexte central européen. Dans ce domaine, elle a coordonné en 2009 le numéro thématique de la revue Synergies Roumanie "Sciences du langage et didactique des langues. Frontières et rencontres" et a publié en 2015 Le plurilinguisme dans la formation universitaire des enseignants de langues. Compte rendu d'une expérience en contexte roumain, Bucuresti : Editura Universitara. En 2020 elle a co-coordonné avec Stéphanie Galligani le numéro 67 de la revue Recherches et Applications « Didactique du plurilinguisme et formation des enseignants : contextes, dispositifs et perspectives $»$.

Courriel : monicavlad[at]yahoo.fr 